\title{
Globalization of the actions of the Lie symmetries of nonlinear wave equations with dissipation
}

\author{
Jose A. Franco ${ }^{1}$
}

Received: 24 September 2014 / Revised: 17 March 2015 / Accepted: 18 March 2015 /

Published online: 1 April 2015

(C) Springer International Publishing AG 2015

\begin{abstract}
The classification of the Lie point symmetries of the nonlinear wave equations with dissipation give a generic case and eight special cases. In this article we show that in seven of the eight cases the action of the Lie symmetries can be globalized via induced representations of a family of solvable Lie groups. In one special case, we show that the action of the Lie symmetries can be globalized by using an induced representation of $\mathrm{GL}(2, R)$.
\end{abstract}

Keywords Globalizations · Solvable Lie groups · Nonlinear wave equation with dissipation · Parabolic induction

Mathematics Subject Classification $\quad 22 \mathrm{E} 25 \cdot 22 \mathrm{E} 27 \cdot 22 \mathrm{E} 46 \cdot 22 \mathrm{E} 70$

\section{Introduction}

Symmetry methods in the analysis of differential equations have been extensively studied. References in the literature abound; the reader can be referred to [1,7-9] for some extensive treatments of the subject. Lie's original prolongation algorithm provides infinitesimal generators for the symmetry groups of differential equations. It is well known that the actions of these generators exponentiate to one-parameter local Lie groups, c.f. [9]. However, these actions seldom exponentiate to a global action of a Lie group. Hence, many of the tools from Lie theory cannot be used. However, in a groundbreaking article Craddock discovered that by restricting to a special subset

$凶$ Jose A. Franco

jose.franco@unf.edu

1 Department of Mathematics and Statistics, University of North Florida, 1 UNF Drive, Building 14E, Jacksonville, FL 32224, USA 
of the solution space of a partial differential equation, the local actions generated by its symmetry Lie algebra could be globalized, c.f. [2]. This started the study of the the globalization problem for the action of symmetry groups of differential equations. In $[5,6,10,13]$, this problem is solved by using the representation theoretic technique of parabolic induction. Using this method, spaces of solutions to the different partial differential equations are constructed. These spaces consist of smooth functions on which the actions of the Lie symmetries globalize. The most refined results are attained when considering linear equations. However, this problem has also been studied for nonlinear equations in $[5,11,12]$. In this article, most of the symmetry groups are solvable. In the special case of nilpotent groups, Craddock and Dooley studied the heat equation on nilpotent groups in [3]. As a result, they studied a very similar equation as the one that concerns us in this article.

Here we consider the globalization problem for the family of nonlinear wave equations with dissipation of the form

$$
u_{t t}+\varphi(u) u_{t}=\left(f(u) u_{x}\right)_{x} .
$$

It is well known [7] that the infinitesimal generators

$$
\left\{X_{1}=\partial_{t}, X_{2}=\partial_{x}\right\}
$$

of the symmetry group form an Abelian Lie algebra. It is also well known that the symmetry algebra extends in five special cases of $f$, and some special cases of $\varphi$. The special cases of $f$ are

$$
\begin{gathered}
f(u)=1, \quad f(u)= \pm e^{u}, \quad f(u)= \pm u^{\sigma}, \quad f(u)= \pm u^{-4}, \\
f(u)= \pm u^{-4 / 3},
\end{gathered}
$$

where $\sigma \in \mathbb{R}^{\times}$. In all these cases, with the exception of a special case (2), the symmetry Lie algebra is extended by an operator of the form

$$
X_{3}=\alpha x \partial_{x}+\beta t \partial_{t}+(\gamma u+\kappa) \partial_{u},
$$

where $\alpha, \beta, \gamma$ and $\kappa$ are constants and $\gamma \cdot \kappa=0$. Following the ideas in $[5,10,11]$, we construct a space of solutions on which the action of a solvable group globalizes the action of the one-parameter subgroups generated by the symmetry group of the corresponding differential equation. In the special case of (2) the Lie algebra spanned by the Lie symmetries is isomorphic to $\mathfrak{s l}(2, \mathbb{R})$ and the analysis is more interesting. In the end, we are able to globalize the action of the symmetry group of each of the special cases of the nonlinear wave equations with dissipation on a space of smooth functions. For more on Lie symmetry analysis of equations with $\mathfrak{s l}(2, \mathbb{R})$-symmetries see [4], where equations with equivalent Lie symmetries are studied.

\section{Solvable group}

To construct a space of functions on which the action of the symmetry group can be globalized we consider the family of solvable Lie groups 


$$
G_{\alpha, \beta}=\left\{g_{q, t, x}=\left(\begin{array}{ccc}
q^{\beta} & 0 & t \\
0 & q^{\alpha} & x \\
0 & 0 & 1
\end{array}\right): t, x \in \mathbb{R}, q \in \mathbb{R}^{>0}\right\}, \quad \alpha, \beta \in \mathbb{R}
$$

and the subgroup

$$
P_{\alpha, \beta}=\left\{g_{q, 0,0}: q \in \mathbb{R}^{>0}\right\}, \quad \alpha, \beta \in \mathbb{R}
$$

On this subgroup, we define the family of characters parametrized by $\gamma \in \mathbb{R}$, $\chi_{\gamma}: P_{\alpha, \beta} \rightarrow \mathbb{C}^{\times}$by

$$
\chi_{\gamma}\left(g_{q, 0,0}\right)=q^{-\gamma}
$$

Then, the induced representation of $G_{\alpha, \beta}$ by this character on $P_{\alpha, \beta}$ is given by

$$
\begin{aligned}
I_{\alpha, \beta, \gamma} & =\operatorname{Ind}_{P}^{G_{\alpha, \beta}}\left(\chi_{\gamma}\right) \\
& =\left\{\varphi \in C^{\infty}\left(G_{\alpha, \beta}\right): \varphi(g p)=\chi(p)^{-1} \varphi(g), g \in G_{\alpha, \beta}, p \in P_{\alpha, \beta}\right\},
\end{aligned}
$$

where the action of $G_{\alpha, \beta}$ on this space is given by left translation.

The subgroup

$$
N_{\alpha, \beta}=\left\{g_{1, t, x}: t, x \in \mathbb{R}\right\}
$$

is canonically isomorphic to $\mathbb{R}^{2}$. Using this isomorphism, we can define a representation of $G_{\alpha, \beta}$ isomorphic to $I_{\alpha, \beta, \gamma}$ by restricting the domain of the maps in $I_{\alpha, \beta, \gamma}$ to $N_{\alpha, \beta}$. More specifically, we define the space

$$
I_{\alpha, \beta, \gamma}^{\prime}=\left\{f \in C^{\infty}\left(\mathbb{R}^{2}\right): f(t, x)=\varphi\left(g_{1, t, x}\right),(t, x) \in \mathbb{R}^{2} \text {, for some } \varphi \in I_{\alpha, \beta, \gamma}\right\} .
$$

To see that this restriction map defines an isomorphism, we notice the easily checked fact that

$$
g_{q, t, x}=g_{1, t, x} \cdot g_{q, 0,0} .
$$

Thus,

$$
\varphi\left(g_{q, t, x}\right)=\varphi\left(g_{1, t, x} \cdot g_{q, 0,0}\right)=q^{\gamma} \varphi\left(g_{0, t, x}\right)=q^{\gamma} f(t, x)
$$

Hence, the value of $\varphi$ at $g_{q, t, x}$ is completely determined by the value of $f(t, x)$. Thus, the restriction map is surjective and it is clearly injective. To see that the isomorphism intertwines with the action of $G_{\alpha, \beta}$ we define the action of $G_{\alpha, \beta}$ on $I_{\alpha, \beta, \gamma}^{\prime}$ by

$$
g_{q_{1}, t_{1}, x_{1}} \cdot f(t, x)=q_{1}^{\gamma} f\left(\left(t-t_{1}\right) q_{1}^{-\beta},\left(x-x_{1}\right) q_{1}^{-\alpha}\right)
$$

and it is routine to check that the vector space isomorphism is indeed intertwining.

At this point, we compare the action of the Lie algebra $\mathfrak{g}_{\alpha, \beta}$ of $G_{\alpha, \beta}$ with the actions of the symmetries of (1). The Lie algebra $\mathfrak{g}_{\alpha, \beta}$ is spanned by the matrices 
$\left\{\xi_{1}=e_{1,4}, \xi_{2}=e_{2,4}, \xi_{3}=e_{2,2}+e_{3,4}\right\}$, where $e_{i, j}$ is the $3 \times 3$ matrix with zeros in all positions except for a one in the $i, j$-th position. Using (3), we can calculate the action of the one-parameter groups generated by $\left\{\xi_{1}, \xi_{2}, \xi_{3}\right\}$. We obtain

$$
\begin{aligned}
& \left(\exp \left(s \xi_{1}\right) \cdot f\right)(t, x)=f(t-s, x) \\
& \left(\exp \left(s \xi_{2}\right) \cdot f\right)(t, x)=f(t, x-s) \\
& \left(\exp \left(s \xi_{3}\right) \cdot f\right)(t, x)=e^{s \gamma} f\left(e^{-s \beta} t, e^{-s \alpha} x\right) .
\end{aligned}
$$

We use these to show the following theorem.

Theorem 2.1 The action of the groups $G_{\alpha, \beta}$ on $I_{\alpha, \beta, \gamma}^{\prime}$ globalizes the action of the one-parameter groups generated by the Lie symmetries of the equation

$$
u_{t t}+\varphi(u) u_{t}=\left(f(u) u_{x}\right)_{x}
$$

in the following cases:

\begin{tabular}{lllll}
\hline$f(u)$ & $\varphi(u)$ & $\alpha$ & $\beta$ & $\gamma$ \\
\hline 1 & $u^{\sigma}$ & 1 & 1 & $-1 / \sigma$ \\
$\pm u^{\sigma}$ & 1 & 1 & 0 & $2 / \sigma$ \\
$\pm u^{\sigma}$ & $u^{v}$ & $1-\sigma /(2 v)$ & 1 & $-1 / v$ \\
$\pm u^{-4 / 3}$ & $u^{\nu}$ & $1+2 /(3 v)$ & 1 & $-1 / v$ \\
\hline
\end{tabular}

where $\sigma \neq-4 / 3$.

Proof To obtain this result one needs to differentiate the actions (4) and note the fact that a vector field of the form

$$
V=\tau(t, x) \frac{\partial}{\partial t}+\xi(t, x) \frac{\partial}{\partial x}+\phi(t, x, v(t, x)) \frac{\partial}{\partial v}
$$

generates a one-parameter group such that

$$
\begin{array}{r}
\left.\frac{d}{d s}\right|_{s=0}(\exp (s V) \cdot f)(t, x)=-\tau(t, x) \frac{\partial f}{\partial t}(t, x)-\xi(t, x) \frac{\partial f}{\partial x}(t, x) \\
+\phi(t, x, f(t, x)),
\end{array}
$$

c.f. [9]. Then a case by case analysis of the table shows that the action of the oneparameter subgroup of $\xi_{i}$ on $I_{\alpha, \beta, \gamma}^{\prime}$ corresponds with the action of $X_{i}$ in each particular case as desired.

So far, we have just considered the cases when $\gamma \neq 0$. Now, let us consider the cases for which the extension has $\kappa \neq 0$ (and correspondingly $\gamma=0$ ). In this case the action of $G_{\alpha, \beta}$ on $I_{\alpha, \beta, 0}^{\prime}$ becomes

$$
g_{q_{1}, t_{1}, x_{1}} \cdot f(t, x)=f\left(\left(t-t_{1}\right) q_{1}^{-\beta},\left(x-x_{1}\right) q_{1}^{-\alpha}\right) .
$$


Unfortunately, this action does not globalize the action of the one-parameter group generated by $X_{3}$ whenever $\kappa \neq 0$. To obtain the desired globalization, we define the $\operatorname{map} \theta_{\kappa}: G_{\alpha, \beta} \times I_{\alpha, \beta, 0}^{\prime} \rightarrow I_{\alpha, \beta, 0}^{\prime}$ by

$$
\theta_{\kappa}\left(g_{q_{1}, t_{1}, x_{1}}\right) \cdot f(t, x)=f(t, x)+\kappa \ln q_{1} .
$$

This map defines an action of $G_{\alpha, \beta}$ on $I_{\alpha, \beta, 0}^{\prime}$, indeed

$$
\begin{aligned}
\left(\theta_{2}\left(g_{q_{1}, t_{1}, x_{1}} \cdot g_{q_{2}, t_{2}, x_{2}}\right) \cdot f\right)(t, x) & =f(t, x)+\kappa \ln q_{1} q_{2} \\
& =f(t, x)+\kappa \ln q_{1}+\kappa \ln q_{2} \\
& =\left(\theta_{2}\left(g_{q_{1}, t_{1}, x_{1}}\right) \cdot\left(\theta_{2}\left(g_{q_{2}, t_{2}, x_{2}}\right) \cdot f\right)\right)(t, x) .
\end{aligned}
$$

Using this, we can define an action $\gamma_{\kappa}: G_{\alpha, \beta} \times I_{\alpha, \beta, 0}^{\prime} \rightarrow I_{\alpha, \beta, 0}^{\prime}$ by

$$
\left(\gamma_{\kappa}(g) \cdot f\right)(t, x)=\left(\theta_{\kappa}(g) \cdot(g \cdot f)\right)(t, x) .
$$

This action can be explicitly calculated; we obtain

$$
\gamma_{\kappa}\left(g_{q_{1}, t_{1}, x_{1}}\right) \cdot f(t, x)=f\left(\left(t-t_{1}\right) q_{1}^{-\beta},\left(x-x_{1}\right) q_{1}^{-\alpha}\right)+\kappa \ln q_{1} .
$$

Now we can calculate the action of the one-parameter groups generated by $\left\{\xi_{1}, \xi_{2}, \xi_{3}\right\}$; we obtain

$$
\begin{aligned}
& \left(\exp \left(s \xi_{1}\right) \cdot f\right)(t, x)=f(t-s, x) \\
& \left(\exp \left(s \xi_{2}\right) \cdot f\right)(t, x)=f(t, x-s) \\
& \left(\exp \left(s \xi_{3}\right) \cdot f\right)(t, x)=f\left(e^{-\beta s} t, e^{-\alpha s} x\right)+\kappa s .
\end{aligned}
$$

Differentiating these actions as in Theorem 2.1, we obtain the following theorem.

Theorem 2.2 The action $\gamma_{\kappa}: G_{\alpha, \beta} \times I_{\alpha, \beta, 0}^{\prime} \rightarrow I_{\alpha, \beta, 0}^{\prime}$ globalizes the action of the oneparameter groups generated by the Lie symmetries of the equation

$$
u_{t t}+\varphi(u) u_{t}=\left(f(u) u_{x}\right)_{x}
$$

in the following cases:

\begin{tabular}{lllll}
\hline$f(u)$ & $\varphi(u)$ & $\alpha$ & $\beta$ & $\gamma$ \\
\hline 1 & $e^{u}$ & 1 & 1 & -1 \\
$\pm e^{u}$ & 1 & 1 & 0 & 2 \\
$\pm e^{u}$ & $e^{k u}$ & $1-1 /(2 k)$ & 1 & $-1 / k$ \\
\hline
\end{tabular}

where $k$ is an arbitrary non-zero constant. 
This completes the globalization problem for the Lie symmetries of the nonlinear wave equations with dissipation for which the Lie symmetries form a solvable Lie algebra.

\section{Case: $f(u)= \pm u^{-4 / 3}$ and $\varphi(u)=1$}

This case is fundamentally different than the previous cases. In particular, because in this case the symmetry Lie algebra is isomorphic to $\mathfrak{g l}(2, \mathbb{R})$, hence reductive; in all previous cases the Lie algebras were solvable.

As it is well known $\mathfrak{g l}(2, \mathbb{R}) \cong \mathfrak{s l}(2, \mathbb{R}) \oplus \mathbb{R}$. In this case, a standard $\mathfrak{s l}(2, \mathbb{R})$-triple is given by the set $\left\{2 x \partial_{x}-3 u \partial_{u}, \partial_{x},-x^{2} \partial_{x}+3 x(u+\mu) \partial_{u}\right\}$ and the operator $\partial_{t}$ spans the center of the Lie algebra. To fully take advantage of the semisimple structure of $\mathfrak{s l}(2, \mathbb{R})$, we will continue to realize $\mathfrak{g l}(2, \mathbb{R})$ as $\mathfrak{s l}(2, \mathbb{R}) \oplus \mathbb{R}$ and the corresponding construction at the level of the groups. So let $G$ be the connected component of $\mathrm{GL}(2, \mathbb{R})$ that contains the identity. Then, $G$ can be realized as the group

$$
G=\left\{\left(\left(\begin{array}{ll}
a & b \\
c & d
\end{array}\right), g\right): a, b, c, d \in \mathbb{R}, g \in \mathbb{R}^{>0}, a d-b c=1\right\}
$$

with the product defined coordinate-wise with matrix multiplication on the first coordinate and scalar multiplication on the second. Consider the parabolic subgroup

$$
P=\left\{\left(\left(\begin{array}{cc}
a & 0 \\
c & a^{-1}
\end{array}\right), 1\right): a, b, c \in \mathbb{R}\right\}
$$

and the subgroup,

$$
N=\left\{N_{t, x}=\left(\left(\begin{array}{ll}
1 & x \\
0 & 1
\end{array}\right), e^{t}\right): t, x \in \mathbb{R}\right\}
$$

From its definition we can see that $N$ is isomorphic to $\mathbb{R}^{2}$ via the map $(t, x) \rightarrow N_{t, x}$.

We will use parabolic induction to construct a representation of $G$ via a character $\chi: P \rightarrow \mathbb{C}^{\times}$that we will define by

$$
\chi\left(\left(\begin{array}{ll}
a & 0 \\
c & a^{-1}
\end{array}\right), 1\right)=a^{-3}
$$

The corresponding induced representation of $G$ is

$$
I=\operatorname{Ind}_{P}^{G}(\chi)=\left\{\varphi \in C^{\infty}(G): \varphi(g p)=\chi(p)^{-1} \varphi(g), g \in G, p \in P\right\}
$$

with the action of $G$ on $I$ given by left translation, that is

$$
g_{1} \cdot \varphi\left(g_{2}\right)=\varphi\left(g_{1}^{-1} g_{2}\right)
$$


An element in $G$ can be decomposed as a product of an element in $N$ times an element in $P$ in the following way:

$$
\left(\left(\begin{array}{ll}
a & b \\
c & d
\end{array}\right), g\right)=\left(\left(\begin{array}{cc}
1 & b d^{-1} \\
0 & 1
\end{array}\right), g\right)\left(\left(\begin{array}{cc}
d^{-1} & 0 \\
c & d
\end{array}\right), 1\right)
$$

provided $d \neq 0$. The set $N P$ forms an open dense set in $G$. Therefore, a function $\varphi \in I$ is completely determined by its values on $N$. This implies that the map $\Xi: I^{\prime} \rightarrow$ $C^{\infty}\left(\mathbb{R}^{2}\right)$ given by $\Xi(\varphi)(t, x)=\varphi\left(N_{t, x}\right)$ is injective. Let $I^{\prime}$ be the image of $\Xi$ in $C^{\infty}\left(\mathbb{R}^{2}\right)$, that is

$$
I^{\prime}=\left\{f \in C^{\infty}\left(\mathbb{R}^{2}\right): f(t, x)=\varphi\left(N_{t, x}\right),(t, x) \in \mathbb{R}^{2}, \text { for some } \varphi \in I\right\} .
$$

Since $\Xi$ is certainly linear, it is a vector space isomorphism between $I$ and $I^{\prime}$. The space $I^{\prime}$ can be given the structure of a $G$ module such that the restriction map $\varphi \mapsto f$ is intertwining. This action can be obtained explicitly as follows

$$
\begin{aligned}
\left(\left(\left(\begin{array}{ll}
a & b \\
c & d
\end{array}\right), g\right) \cdot f\right) & (t, x)=\left(\left(\left(\begin{array}{ll}
a & b \\
c & d
\end{array}\right), g\right) \cdot \varphi\right)\left(N_{t, x}\right) \\
& =\varphi\left(\left(\left(\begin{array}{ll}
a & b \\
c & d
\end{array}\right), g\right)^{-1} \cdot N_{t, x}\right) \\
& =\varphi\left(\left(\left(\begin{array}{cc}
d & d x-b \\
-c & a-c x
\end{array}\right), g^{-1} e^{t}\right)\right) \\
& =(a-c x)^{-3} \varphi\left(\left(\left(\begin{array}{l}
1 \\
0
\end{array}\right)\right.\right. \\
& =(a-c x)^{-3} f\left(t-\ln g, \frac{d x-b}{a-c x}\right) .
\end{aligned}
$$

By differentiating the action (5), we obtain the following theorem.

Proposition 3.1 An element $\left(\left(\begin{array}{cc}\alpha & \beta \\ \gamma & -\alpha\end{array}\right), \delta\right) \in \mathfrak{s l}(2, \mathbb{R}) \oplus \mathbb{R}$ acts on $I^{\prime}$ by

$$
3(x \gamma-\alpha) u \partial_{u}-\delta \partial_{t}+\left(\gamma x^{2}-\beta-2 \alpha\right) \partial_{x} .
$$

In particular, the action (5) of $G$ on $I^{\prime}$ globalizes the action of the one-parameter groups generated by the Lie symmetries of the equation

$$
u_{t t}+u_{t}=\left( \pm u^{-4 / 3} u_{x}\right)_{x}
$$

\section{References}

1. Bluman, G.W., Anco, S.C.: Symmetry and Integration Methods for Differential Equations. Applied Mathematical Sciences, vol. 154. Springer, New York (2002)

2. Craddock, M.: The symmetry groups of linear partial differential equations and representation theory. I. J. Differ. Equ. 116(1), 202-247 (1995) 
3. Craddock, M.J., Dooley, A.H.: Symmetry group methods for heat kernels. J. Math. Phys. 42(1), 390-418 (2001)

4. Craddock, M.J., Dooley, A.H.: On the equivalence of Lie symmetries and group representations. J. Differ. Equ. 249(3), 621-653 (2010)

5. Franco, J.A.: Global $\widehat{\operatorname{SL}(2, R)}$ representations of the Schrödinger equation with singular potential. Cent. Eur. J. Math 10(3), 927-941 (2012)

6. Franco, J.A., Sepanski, M.R.: Global representations of the heat and Schrödinger equation with singular potential. Electron. J. Differ. Equ. N154 (2013)

7. Ibragimov, N.H. (ed.): CRC Handbook of Lie Group Analysis of Differential Equations. I. CRC Press, Boca Raton (1994)

8. Ibragimov, N.H. (ed.): CRC Handbook of Lie Group Analysis of Differential Equations. II. CRC Press, Boca Raton (1995)

9. Olver, P.J.: Applications of Lie Groups to Differential Equations. Graduate Texts in Mathematics, vol. 107, 2nd edn. Springer, Berlin (2000)

10. Sepanski, M.R.: Global actions of Lie symmetries for the nonlinear heat equation. J. Math. Anal. Appl. 360(1), 35-46 (2009)

11. Sepanski, M.R.: Nonlinear potential filtration equation and global actions of Lie symmetries. Electron. J. Differ. Equ. N101 (2009)

12. Sepanski, M.R., Stanke, R.J.: On global SL(2, $\mathbb{R})$ symmetries of differential operators. J. Funct. Anal. 224(1), 1-21 (2005)

13. Sepanski, M.R., Stanke, R.J.: Global Lie symmetries of the heat and Schrödinger equation. J. Lie Theory 20(3), 543-580 (2010) 